\title{
Estudio del comportamiento de la albañilería confinada en el análisis estático y dinámico para la ciudad de Potosí, Bolivia
}

Study of behavior of the masonry confined in the static and dynamic analysis for the city of Potosí, Bolivia

Gabriela González, rrppuatf@gmail.com Universidad Autónoma Tomás Frías, Bolivia

\section{RESUMEN}

El presente trabajo tuvo como fin estudiar el comportamiento estático y dinámico de la albañilería confinada. Para ello se considera una edificación que cumpla las características de simetría en planta como en elevación. Ésta fue modelada mediante el programa ETABS para su análisis estructural. Previa a la modelación se realizan ensayos para conocer características físico-mecánicas que presentan los ladrillos Dipafex usualmente utilizados en la construcción en la ciudad de Potosí.

Palabras clave: Albañilería Confinada; diseño sismoresistente; Ladrillos Dipafex

\section{ABSTRACT}

The purpose of this work was to study the static and dynamic behavior of confined masonry. This is considered a building that meets the characteristics of symmetry in the plant and elevation. This was modeled through the ETABS program for structural analysis. Prior to modeling, tests are carried out to know the physical-mechanical characteristics of the Dipafex bricks usually used in construction in the city of Potosí.

Key words: Confined Masonry; earthquake resistant design; Dipafex Bricks 


\section{INTRODUCCIÓN}

Las estructuras en general se ven amenazadas por eventos sísmicos. López et al. (2017) expresan que estos se presentan en todo el mundo, en algunas regiones más que en otras. Sobre este amplio tema también conviene resaltar lo manifestado por Salamanca et al. (2011); Grandi (2006) y Albariño et al. (2002) sobre la historia de seísmos y sus consecuencias, pues Bolivia se ubica en la región conocida como "Cinturón de Fuego" del Pacífico, llamada así por la intensa actividad sísmica que presenta la zona. Este hecho obligó a hacer un rediseño de las estructuras para que estas puedan comportarse adecuadamente o, por lo menos, no colapsar al ser sometida a estos eventos dinámicos.

Es así que se trataron de adecuar y efectivizar los modelos estructurales ya conocidos, uno de estos, expresan Valdebenito et al. (2015) fueron las estructuras de albañilería, donde el principal elemento estructural es la misma, que presenta buen comportamiento estático, pero sometida a eventos sísmicos este tipo de estructuras son muy frágiles. Para hacer más efectivo la albañilería se procedió, tomando en cuenta lo manifestado por Astroza (2004) a confinarlas con un marco de hormigón origi-nados así las estructuras de Albañilería confinada.

Para San Bartolomé et al. (2011) la albañilería o mampostería se define como un conjunto de unidades de ladrillo trabadas o adheridas entre sí con algún material. La albañilería confinada es simplemente estas piezas de ladrillo adheridas y confinados con columnas y vigas cadena de hormigón.

Este sistema fue creado por el hombre a fin de satisfacer sus necesidades principalmente de vivienda, es así que la albañilería confinada por elementos de concreto armado fue creada por ingenieros italianos, después que el sismo ocurrido en 1908 en Messina, Sicilia arrasara con las edificaciones de albañilería no reforzada (San Bartolomé, 1994, s/n).

La albañilería fue introducida en Bolivia tan solo con fines constructivos, como tabiques separador de ambientes, pero dejando de lado el comportamiento estructural que este sistema tiene. En algunos sectores de la ciudad, comenta Aguirre (2013), se realizan construcciones de viviendas pequeñas de albañilería, esta trabaja como muro portante, y en otros casos realizan la construcción de los muros con el proceso constructivo de la albañilería confinada, es decir primero los muros, después las columnas y vigas. Esto para ahorrar material de encofrado o simplemente como una técnica constructiva más. En otras palabras, el uso de la albañilería ya sea simple o confinada es muy usual, pero este uso es empírico, es decir sin conocer el comportamiento y las características físico-mecánicas que la albañilería presenta y más aún cuando esta es confinada.

Para cerrar estas líneas introductorias, se concreta que el presente trabajo se enfoca en analizar el comporta-miento estático y dinámico de la albañilería confinada, considerando métodos numéricos o de elementos finitos y así poder aprovechar la capacidad de resis-tencia que nos ofrece este sistema estructural.

MATERIALES Y MÉTODOS

Este trabajo abarcó varios métodos de investigación: el empírico, estadístico 
matemático y teórico. A continuación una breve explicación de cada uno:

\section{Método empírico}

Con la finalidad de conocer las características de resistencia de la albañilería se realizaron pruebas de laboratorio. La población fue conformada por los cinco (5) muros pequeños alrededor de $50 \times 50 \mathrm{~cm}$ para determinar la resistencia compresión diagonal. Adicionalmente, se usaron otros diez (10) muros para determinar la resistencia a compresión pura.

\section{Métodos del nivel estadístico mate- mático}

Estos métodos se emplearon con la finalidad de analizar cuantitativamente los datos obtenidos en las pruebas indicadas anteriormente y así obtener valores sintetizados y representativos de todas las pruebas.

\section{Métodos de nivel teórico}

Con los métodos teóricos se analizaron los diferentes resultados obtenidos en las pruebas y luego se sintetizaron los resultados de estos ensayos. Por otro lado, también se analizó el comportamiento estático y dinámico de la estructura de albañilería confinada.

\section{Método de modelación}

Se empleó con finalidad de poder realizar un estudio al comportamiento de la albañilería confinada a través del programa ETABS. Cueva et al. (2013) proporcionaron aportes para abordar este método.

\section{Observación}

Se realizó una observación detallada de los resultados obtenidos en las pruebas de laboratorio y en el modelo para conocer el comportamiento de la albañilería.

\section{Novedad científica}

La albañilería confinada es parte del diseño estructural estático y sísmico de otros países, pero en nuestro país no se realiza este tipo de estructuras, quizá debido a que no se cuenta con muchos datos referentes de su comportamiento ni se conoce las características resistentes de los materiales usados, además de no contar con los laboratorios adecuados para realizar un estudio completo y profundo.

En este entendido, este trabajo se enfocó en realizar un estudio del comportamiento de la albañilería confinada sometido a cargas estáticas y sísmicas de manera más simple y por medio de modelaciones computacionales, además de conocer las propiedades físicas $\mathrm{y}$ mecánicas que esta nos presenta.

\section{RESULTADOS Y DISCUSIÓN}

Para realizar un análisis del comportamiento y de las fuerzas internas de la albañilería confinada, en primera instancia se analizaron las características físico mecánicas de acuerdo a la NTP 399.621 (Normas para Muretes de Albañilería), referenciado por Gamarra et al (2018) y NTP 399.613 (Normas de Unidades), revisado por Durand y Benites (2018). Ambos son estudios referenciales de primera fuente que orientan este tipo de investigaciones.

Una vez conocidas estas características consideraremos una estructura de acuerdo a los especificados en la norma E.030 "Diseño sismoresistente", referenciada por San Bartolomé (2008). Una estructura simétrica tanto en planta como en elevación, con formas adecuadas que permitan un buen comportamiento sísmico, además de contar con una 
densidad de muros adecuada. Esta estructura fue modelada usando métodos números "elementos finitos" y métodos manuales que nos ayudaron a entender cómo reacciona la estructura de albañilería confinada ante eventos sísmicos.
Propiedades físicas y mecánicas. Ensayos de laboratorios

Las Tablas del 1 al 4 muestran, tomando en cuenta a González (2017) los resultados de compresión pura y de ladrillos de 18 huecos.

Tabla 1. Resultados del ensayo de compresión

\begin{tabular}{ccccccc}
\hline $\begin{array}{c}\text { NOMBRE } \\
\text { PRISMA }\end{array}$ & Lm (cm) & Am (cm) & Hm (cm) & $\begin{array}{c}\text { Área } \\
\text { (cm2) }\end{array}$ & P (kg) & $\begin{array}{c}\text { fm (kg/ } \\
\mathbf{c m}^{2} \text { ) }\end{array}$ \\
\hline PR 18 - 1 & 24.5 & 11.6 & 38.3 & 283.0 & 43200 & 152.664 \\
PR 18 - 2 & 24.5 & 11.5 & 38.3 & 281.2 & 39300 & 139.771 \\
PR 18 - 3 & 24.6 & 11.5 & 38.5 & 281.1 & 33100 & 117.753 \\
PR 18 - 4 & 24.5 & 11.3 & 38.3 & 276.3 & 44200 & 159.980 \\
PR 18 - 5 & 24.4 & 11.4 & 38.8 & 278.2 & 39700 & 142.724 \\
& & & & & Promedio & 129.746 \\
\hline
\end{tabular}

Tabla 2. Valores promedio

\begin{tabular}{lc}
\hline Promedio & $129.746 \mathrm{~kg} / \mathrm{cm}^{2}$ \\
\hline Desviación Estándar & 13.053 \\
Resistencia característica a compresión fm & $116.693 \mathrm{~kg} / \mathrm{cm}^{2}$ \\
\hline
\end{tabular}

Tabla 3. Prismas de ladrillos de 18 huecos

\begin{tabular}{ccccccc}
\hline $\begin{array}{c}\text { NOMBRE } \\
\text { PRISMA }\end{array}$ & D (cm) & Área (cm2) & P (kg) & $\begin{array}{c}\text { Vm } \\
(\mathbf{k g} / \mathbf{c m} 2)\end{array}$ & $\mathbf{x}-\mathbf{X}$ & $\mathbf{( x - X ) ^ { 2 }}$ \\
\hline PR 18 - 1 & 54.294 & 610.8 & 11.200 & 18.336 & 2,078 & 4,319 \\
PR 18 - 2 & 54.500 & 621.3 & 9.300 & 14.969 & -1.281 & 1,662 \\
PR 18 - 3 & 55.174 & 631.7 & 10.000 & 15.829 & $-0,429$ & 0,184 \\
PR 18 - 4 & 55.596 & 639.4 & 9.800 & 15.328 & -0.930 & 0,865 \\
PR 18 - 5 & 55.015 & 629.9 & 10.600 & 16.828 & 0.570 & $\mathbf{0 , 3 2 5}$ \\
\hline
\end{tabular}

Tabla 4. Valores promedio

\begin{tabular}{lc}
\hline Promedio & $16.258 \mathrm{~kg} / \mathrm{cm}^{2}$ \\
\hline Desviación Estándar & 1.213 \\
Resistencia característica diagonal fm & $15.045 \mathrm{~kg} / \mathrm{cm}^{2}$ \\
\hline
\end{tabular}

\section{Análisis dinámico estructural}

Una vez conocidas las características físico-mecánicas de la albañilería, se usaron estos resultados para realizar el estudio de la albañilería confinada en una vivienda acorde con las especificaciones de la norma.

\section{Descripción de la estructura}

Se ha establecido un edificio de estructura regular destinada a ser una vivienda multifamiliar, el cual cuenta con cinco (5) niveles, siendo el último piso la azotea de uso común. Se trata de una construcción de albañilería confinada, con 
un sistema de techado de losa aligerada unidireccional cuyo espesor es $20 \mathrm{~cm}$ y separación de entre ejes de $50 \mathrm{~cm}$. La altura de piso a techo es de $2.60 \mathrm{~m}$, ancho de puertas $1 \mathrm{~m}$, ancho de las ventanas $1.50 \mathrm{~m}$, peralte viga solera $0.20 \mathrm{~m}$ y peralte de la viga dintel $0.30 \mathrm{~m}$.

\section{Características de los materiales}

\section{Albañilería}

- Ladrillos de arcilla: Dipafex espesor $\mathrm{t}=$ $12 \mathrm{~cm}$.

- Mortero: tipo P1 cemento arena 1:3

- Pilas: resistencia característica a compresión f' $\mathrm{m}=110 \mathrm{~kg} / \mathrm{cm}^{2}$

- Muretes: resistencia característica a corte puro v'm $=15 \mathrm{~kg} / \mathrm{cm}^{2}$

- Módulo de elasticidad $\mathrm{Em}=500 \mathrm{f}^{\prime} \mathrm{m}=$ $55000 \mathrm{~kg} / \mathrm{cm}^{2}$

- Módulo de corte $\mathrm{Gm}=0.4 \mathrm{Em}=22000$ $\mathrm{kg} / \mathrm{cm}^{2}$

- Módulo de Poisson $n=0.25$

\section{Concreto}

- Resistencia nominal a compresión $\mathrm{f}^{\prime} \mathrm{c}$ $=210 \mathrm{~kg} / \mathrm{cm}^{2}$

- Módulo de elasticidad $=\mathrm{Ec}=15000^{*}$ $\operatorname{SQR}(210)=217370.6512 \mathrm{~kg} / \mathrm{cm}^{2}$

- Módulo de Poisson $n=0.15$

\section{Cargas unitarias}

\section{Pesos volumétricos}

- Peso volumétrico del concreto armado: 2.4 ton $/ \mathrm{m}^{3}$

- Peso volumétrico de la albañilería: 1.9 ton $/ \mathrm{m}^{3}$

- Peso unitario del acabado: 2.0 ton $/ \mathrm{m}^{3}$

\section{Carga en cada losa}

- Contra piso $80 \mathrm{~kg} / \mathrm{cm}^{2}$

- Acabado de piso $40 \mathrm{~kg} / \mathrm{cm}^{2}$

- Acabado de techo $40 \mathrm{~kg} / \mathrm{cm}^{2}$
Carga muerta no estructural $\mathrm{DNE}=160 \mathrm{~kg} / \mathrm{m}^{2}$

- Peso propio de la losa maciza de 12 $\mathrm{cm} 0.12 \times 2400=288 \mathrm{~kg} / \mathrm{m}^{2}$

\section{Carga muerta por peso propio D} $=200 \mathrm{~kg} / \mathrm{m} 2$

- Sobrecarga (incluso en escalera): 200 $\mathrm{kg} / \mathrm{m}^{2}$, excepto en azotea: $150 \mathrm{~kg} / \mathrm{m}^{2}$

\section{Carga viva 200 kg/m2}

\section{Muros}

- Peso de los muros de albañilería con 1 cm de acabado: $1.9 \times 0.12+2.0 \times 0.02$

$=0.268$ ton $/ \mathrm{m} 2$

- Ventanas: 0.02 ton $/ \mathrm{m} 2$

\section{Análisis Estático o de Fuerzas Estáticas Equivalentes}

Usualmente, las edificaciones de albañilería son de mediana altura (hasta de 5 pisos), muy rígidas y califican como regulares, por lo que para estas situaciones es suficiente con realizar un análisis sísmico estático, donde la determinación de la fuerza sísmica o cortante basal "V", en las direcciones principales de la edificación está dada por (Norma E.030): $V=Z U S$ $V R P$.

Los factores $\mathrm{Z}=$ zona, $\mathrm{U}=$ uso, y $\mathrm{S}=$ suelo, aparecen en la Norma sísmica E.030. Los términos $\mathrm{C}$, $\mathrm{P}$ y $\mathrm{R}$ fueron tratados para el caso específico de edificios de albañilería.

Determinaremos cada uno de estos parámetros:

Para el factor de zona Z se tomó como base la propuesta normativa del Ingeniero Grandi (2006) que nos presenta un mapa de zonificación sísmica de Bolivia, en este podemos observar que el departamento de Potosí se encuentra en la zona sísmica 1 y 2 , con una aceleración de 0.05 y 0.1 . 


\section{Análisis manual}

El análisis manual es un método aproximado para la determinación de las fuerzas internas en los muros, debido a que no se consideran la influencia de las vigas dinteles y de otro elementos.

La realización de este análisis nos mostró claramente la manera cómo la cortante basal es absorbida por la albañilería de acuerdo a las características del mismo.

\section{Metrado de cargas}

Las cargas actuantes en cada muro se obtienen sumando las cargas directas (peso propio, peso de soleras, dinteles, ventanas y alféizares) más las cargas indirectas (provenientes de la losa del techo: peso propio, acabados y sobrecarga).

\section{Método de análisis mediante elementos finitos}

Con la técnica de elementos finitos pueden modelarse estructuras complejas, además de obtener resultados muy precisos.

Señala Lizarza (2000) que todos los productos Computer and Structures (CSI) trabajan mediante el Método de Elementos Finitos (MEF), originalmente introducido por Turner en 1956, que es una técnica computacional para soluciones aproximadas de una variedad de problemas de ingeniería del mundo real que tienen complejo dominio sujeto a condiciones generales de borde.

Para realizar un análisis de fuerzas en muros estructurales tanto ETABS, referenciado por Cueva et al. (2013) y Lema (2013), como SAP2000, valorado por Lavado (2013) e Hilarión (2014), los cuales trabajan, según Flores y Onate (2011), a través de elementos tipo superficie denominado Elemento Shell (Shell Element).

\section{Consideraciones de la discusión}

- Se ha establecido un edificio de estructura regular destinada a ser una vivienda multifamiliar, el cual cuenta con cinco (5) niveles, siendo el último piso la azotea de uso común.

- Se trata de una construcción de albañilería confinada, con un sistema de techado de losa maciza de espesor es 12 cm.

- La altura de piso a techo es de $2.60 \mathrm{~m}$, ancho de puertas $1 \mathrm{~m}$, ancho de las ventanas $1.50 \mathrm{~m}$, peralte viga solera $0.20 \mathrm{~m}$ y peralte de la viga dintel $0.30 \mathrm{~m}$.

\section{CONCLUSIONES}

Se realizaron ensayos en las unidades de albañilería de 18 huecos de acuerdo con lo establecido en la Norma E.070, obteniendo como resultado final: la resistencia característica a compresión (f'm) de $116.693 \mathrm{~kg} / \mathrm{cm}^{2}$, y la resistencia característica a compresión diagonal ( $\left.\mathrm{v}^{\prime} \mathrm{m}\right)$ de $15.045 \mathrm{~kg} / \mathrm{cm}^{2}$.

Estos resultados nos muestran que la albañilería en la ciudad de Potosí es de buena calidad y alcanza valores satisfactorios, siempre y cuando se cumpla con lo especificado en la norma. Entonces es posible realizar edificaciones de mediana altura con albañilería confinada en nuestra ciudad.

Se realizó el modelo estático de una edificación en base a albañilería confinada en el programa ETABS cuyos resultados mostraron que tanto las columnas como los muros absorben las fuerzas axiales, pero la cantidad de fuerza axial que cada uno de ellos absorbe del total, depende principalmente de las secciones que el 
muro y sus columnas de confinamiento presentan.

También se elaboró el modelo dinámico de la edificación de albañilería confinada del cual se concluye que el sismo provoca fuerzas cortantes a la albañilería confinada siendo esta absorbida por el muro específicamente y no así por las columnas. Esta fuerza cortante genera esfuerzos de tracción y compresión horizontales en el muro, las cuales al sobrepasar la resistencia de corte del muro (vm) provocan fisuras o fallas por corte.

Adicionalmente, se pudo observar que la falla también depende de geometría del muro. Si la longitud es parecida a la altura, entonces se produce una falla por corte, pero si la longitud mucho más grande que la altura el muro tiende a fallar por deslizamiento.

El sismo también provoca momentos flectores en los muros y se pudo observar que este momento induce fuerzas verticales de tracción y compresión en las piezas, originando así las falla por flexión. Las columnas al estar adheridas al muro no presenta momentos flectores pero si esfuerzos de tracción y compresión.

En conclusión, se puede decir que la albañilería por sí sola, presenta buen comportamiento en un análisis estático, pero en un análisis dinámico la albañilería necesita refuerzo porque esta no puede absorber los esfuerzos axiales de tracción siendo un complemento efectivo confinarlas en columnas y vigas de concreto.

\section{REFERENCIAS}

Aguirre, S. C. (2013). Boom en el sector inmobiliario en Bolivia: ¿burbuja o fundamentos económicos?

Albariño, L., Dalenz Farjat, A., Alvarez, L., Hernandez, R., \& Pérez Leyton, M. (2002). Las Secuencias Sedimentarias del Devónico en el Subandino Sur y el Chaco. Bolivia y Argentina. In
Congreso de Exploración y Desarrollo de Hidrocarburos (No. 5).

Astroza, M., y Schmidt, A. (2004). Capacidad de deformación de muros de albañilería confinada para distintos niveles de desempeño. Revista de Ingeniería Sísmica, (70), 59-75

Cueva Jiménez, R. A., Chalcuacán, G., y Xavier, D. (2013). Diseño por desempeño de edificaciones en homigón armado con muros de corte mediante los códigos FEMA, utilizando el programa ETABS

Durand, R., y Benites, L. (2018). Unidades de albañilería fabricadas con suelocemento como alternativa para la construcción sostenible. Revista Ciencia y tecnología, 13(1), 21-32

Flores, F. G., y Onate, E. (2011). Un elemento de sólido con una mejora en el comportamiento del corte transversal para el tratamiento de láminas. Revista Internacional de Métodos Numéricos para Cálculo y Diseño en Ingeniería, 27(4), 258-268

Gamarra Cotohuanca, N., Galiano, M., y Luis, J. (2018). Evaluación comparativa de la resistencia a la compresión axial y diagonal en muros de albañilería elaborados con un mortero convencional (C: A) 1: 4, respecto a muros de albañilería elaborados con un mortero adicionado con fibras de polipropileno, utilizando ladrillo king kong 18 huecos

González, G. (2017) Estudio del Comportamiento de la albañilería confinada en el Análisis Estático y Dinámico para la ciudad de Potosí. Universidad Autónoma "Tomás Frías", Potosí, Bolivia

Grandi, R. (2006). Norma Boliviana de Diseño Sísmico. Primera Edición. Ministerio de Servicios y Obras Públicas. Bolivia

Hilarión, F. L. N., y Segrera, D. L. L. (2014). Programa didáctico a código abierto de análisis dinámico de estructuras UNDIN 1.0. Revista Educación en Ingeniería, 9(17), 118-131 
Lavado Rodríguez, J., y Granados Romera, J. J. (2013). Cálculo de estructuras con el programa SAP 2000

Lema Toapanta, E. P. (2013). Análisis y diseño de un edificio con aisladores sísmicos modelamiento en el ETABS

Lizarza, J. T. C. (2000). Método de los elementos finitos para análisis estructural. Escuela Superior de Ingeniros Industriales, Universidad de Navarra

López, A., Álvarez, C. I., y Villarreal, E. (2017). Migración de fuentes sísmicas a lo largo del cinturón de fuego del Pacífico. La Granja. Revista de Ciencias de la Vida, 25(1), 5-15

Salamanca Mazuelo, L., Quiroga Becerra de la Roca, R., y Zamora Auza, B. (2011). Ochenta y cinco años de la historia de desastres en Bolivia (1920-2005). Revista Virtual REDESMA, 5, 15
San Bartolomé, Á. (1994). Construcciones de Albañilería. Fondo editorial de Universidad Católica Perú

San Bartolomé, A. (2008). Comentarios a la norma técnica de edificación E. 070. Perú: Sencico

San Bartolomé, Á. (2011) Diseño y construcción de estructuras sismorresistentes de albañilería. Fondo editorial de La Pontificia Universidad Católica del Perú ed. San Bartolomé Á, Quiun D, Silva W, editores. Lima: Fondo editorial de La Pontificia Universidad Católica del Perú

Valdebenito, G., Alvarado, D., Sandoval, C., y Aguilar, V. (2015, Marzo). Terremoto de Iquique $\mathrm{Mw}=8,2-01$ abril 2014: daños observados y efectos de sitio en estructuras de albañilería. En XI Congreso Chileno de Sismología e Ingeniería Sísmica (pp. 18-20) 